\title{
AN IDENTITY ON ALGEBRAS OVER A HOPF ALGEBRA
}

\author{
STAVROS PAPASTAVRIDIS
}

ABSTRACT. Let $A$ be a connected Hopf algebra which has an associative comultiplication $\psi: A \rightarrow A \otimes A$. Let $\chi: A \rightarrow A$ be the canonical conjugation on $A$. Let $M$ be a graded algebra over the Hopf algebra $A$. If $x, y \in M$, $\psi(a)=\Sigma a^{\prime} \otimes a^{\prime \prime}$, then we have the identity

$$
a x \cdot y=\Sigma(-1)^{\operatorname{deg} x \cdot \operatorname{deg} a^{\prime \prime}} a^{\prime}\left(x \cdot \chi\left(a^{\prime \prime}\right) y\right) .
$$

If $X$ is a topological space and $x, y \in H^{*}\left(X ; Z_{2}\right)$, then we have the identity

$$
\mathrm{Sq}^{n} x \cdot y=\sum_{i=0}^{n} \mathrm{Sq}^{i}\left(x \cdot \chi\left(\mathrm{Sq}^{n-i}\right) y\right)
$$

(for the proof see [1]). This formula has been proved useful in quite a few occasions (for example see [1], [2]).

In this note we give a generalization of this formula for algebras over a Hopf algebra.

Let $A$ be a Hopf algebra over a commutative ring with unit. We assume that $A$ is connected and that its comultiplication $\psi$ is associative. Let $\chi$ : $A \rightarrow A$ be the canonical conjugation of $A$. Let $M$ be a graded algebra over the Hopf algebra $A$. For the terminology and the basic results, we refer to [3]. Under those assumptions, we have

THEOREM. Let $x, y \in M$ and $\psi(a)=\sum_{i} a_{i}^{\prime} \otimes a_{i}^{\prime \prime}$. Then we have

$$
a x \cdot y=\sum_{i}(-1)^{\operatorname{deg} x \cdot \operatorname{deg} a_{i}^{\prime \prime}} a_{i}^{\prime}\left(x \cdot \chi\left(a_{i}^{\prime \prime}\right) y\right) \text {. }
$$

Proof. Let

$$
\psi\left(a_{i}^{\prime}\right)=\sum_{j} b_{i j}^{\prime} \otimes b_{i j}^{\prime \prime} \quad \text { and } \quad \psi\left(a_{i}^{\prime \prime}\right)=\sum_{j} c_{i j}^{\prime} \otimes c_{i j}^{\prime \prime} .
$$

Since $\psi$ is associative, we will have

$$
\sum_{i, j} b_{i j}^{\prime} \otimes b_{i j}^{\prime \prime} \otimes a_{i}^{\prime \prime}=\sum_{i, j} a_{i j}^{\prime} \otimes c_{i j}^{\prime} \otimes c_{i j}^{\prime \prime} .
$$

From the basic properties of tensor products and the fact that $\chi$ is linear, we get that

$$
\sum_{i, j} b_{i j}^{\prime} \otimes b_{i j}^{\prime \prime} \cdot \chi\left(a_{i}^{\prime \prime}\right)=\sum_{i, j} a_{i}^{\prime} \otimes c_{i j}^{\prime} \cdot \chi\left(c_{i j}^{\prime \prime}\right)
$$

Received by the editors June 28, 1977.

AMS (MOS) subject classifications (1970). Primary 57F05.

Key words and phrases. Hopf algebras. 
So we get (see [1, p. 171])

$$
\begin{aligned}
\sum_{i, j}(-1)^{\operatorname{deg} x \cdot \operatorname{deg} b_{i j}^{n}} b_{i j}^{\prime} x \cdot(-1)^{\operatorname{deg} x \cdot \operatorname{deg} a_{i j}^{\prime \prime}} b_{i j}^{\prime \prime} \chi\left(a_{i}^{\prime \prime}\right) y \\
=\sum_{i}(-1)^{\operatorname{deg} x \cdot \operatorname{deg} a_{i}^{\prime \prime}} a_{i}^{\prime} x \cdot\left(\sum_{j} c_{i j}^{\prime} \chi\left(c_{i j}^{\prime \prime}\right)\right) y .
\end{aligned}
$$

But $\Sigma_{j} c_{i j}^{\prime} \chi\left(c_{i j}^{\prime \prime}\right)=0$ unless $\operatorname{deg} a_{i}^{\prime \prime}=0$, so the second member of the previous identity is $a x \cdot y$. Because of the "Cartan" formula (see [3, p. 173]), the first member of the previous identity is equal to the second member of the identity that we want to prove. That ends the proof.

\section{REFERENCES}

1. S. Papastavridis, A formula for the obstruction to transversality, Topology 11 (1972), 415-416.

2. _ The Arf invariant of manifolds with few non-zero Stiefel-Whitney classes, $\mathrm{Ph}$. $\mathrm{D}$. Thesis, Princeton Univ., Princeton, N. J., 1974.

3. N. Steenrod, The cohomology algebra of a space, Enseignement Math. 7 (1961), 153-177.

University of Athiens, Mathematical Institute, Solonos 57, Athens 143, Grebce

Current address: Department of Mathematics, University of Crete, Iraklion, Greece 